\section{LA INTEGRACION ECONÓMICA Y LA TRANSFORMACIÓN PRODUCTIVA EN AMÉRICA LATINA}

\section{Dra. BEATRIZ HERRERA GARCÍA}

En 1990 concluyó la década de la gran crisis del desarrollo latinoamericano. Esta dejó entre sus muchos legados la profundización del conocimiento en los ámbitos de la gestión macroeconómica y de la modernización productiva, pero, como contrapartida, un importante cúmulo de rezagos sociales que afectan a una proporción significativa de la población latinoamericana.

Hoy entendemos mejor que antes los elementos centrales de una buena gestión macroeconómica, el efecto regresivo de un ajuste desordenado y recesivo, la importancia de la política microeconómica, el vínculo entre el desarrollo y el medio ambiente, así como la naturaleza de la innovación y su aplicación al proceso productivo. También se ha profundizado en el análisis de las complementariedades y las oposiciones entre políticas que buscan el crecimiento y aquellas centradas en la equidad.

Por otro lado, el panorama económico de la región ha cambiado considerablemente en los últimos años. En distinto grado entre un país y otro, los desequilibrios macroeconómicos característicos del decenio anterior comenzaron a ceder; la transferencia negativa de recursos financieros se convirtió en una afluencia neta de capitales externos; el sector exportador creció y se diversificó, y la actividad económica en su conjunto exhibió un modesto repunte sobre bases cualitativamente distintas de las que existían hace apenas unos años. Así, el ambiente de abatimiento de los años ochenta ha dado paso a la efervescencia de los noventa.

La interacción dinámica de la región con el resto del mundo se puede dar por dos vectores; primero, la interacción de cada país latinoamericano con los demás en el ámbito de la integración regional $y$, segundo, la interacción de cada uno de ellos con la economía extrarregional. Ambos temas están íntimamente interrelacionados, ya que los compromisos integradores contemporáneos pueden y deben contribuir a mejorar la inserción internacional de los países de la región, a la vez que una mayor participación en la economía internacional tendrá que fortalecer los vínculos intrarregionales de interdependencia económica.

En los últimos años, frente a los efectos de la prolongada crisis de los años ochenta y a las transformaciones en el panorama internacional. América Latina ha demostrado creciente capacidad de respuesta buscando nuevas formas de adaptarse a las cambiantes circunstancias para enfrentar los múltiples desafíos que se le plantean.

El renovado interés en los acuerdos formales de integración económica es un fenómeno atribuible a varios factores, entre los que cabe mencionar el perfeccionamiento de la Unión Europea, la operatividad del tratado de Libre Comercio, la gradual convergencia de las políticas económicas 
aplicadas en la región y la creciente afinidad política entre los gobiernos democráticos. Todo lo anterior persuade acerca las bondades de la integración económica y de la formulación de políticas más apropiadas para una transformación productiva con desarrollo y bienestar generalizados.

La integración económica persigue fortalecer los vínculos recíprocos entre la apertura comercial y las políticas de desregulación, y la tendencia "natural" no discriminatoria frente a terceros países, bajo un marco de relaciones económicas regionales abiertas, es decir, un proceso de creciente interdependencia económica a nivel regional, impulsado tanto por acuerdos preferenciales de integración como por otras políticas en un contexto de apertura y desregulación, con el objeto de aumentar la competitividad de los países de la región y de constituir, en lo posible, un cimiento para una economía mundial más abierta y transparente. Con todo, de no producirse ese escenario óptimo, las relaciones económicas regionales abiertas de todas maneras cumplirían una función importante, en este caso serviría como un mecanismo de defensa ante los efectos de eventuales presiones proteccionistas en mercados extrarregionales.

\section{1.- LA ESTABILIZACIÓN ECONOMICA Y EL FINANCIAMIENTO EXTERNO}

\subsection{El apoyo a las balanzas de pagos.-} Para conjugar efectivamente el riesgo a nivel de país que surge de los desequilibrios macroeconómicos transitorios se requiere, entre otras cosas, del apoyo a las balanzas de pagos. Como la experiencia de la década anterior lo demuestra, cuando en los países de la región se registran déficit globales en la balanza de pagos, el comercio intrarregional también resulta afectado, a pesar de no constituir la principal fuente del déficit. En consecuencia, los mecanismos regionales de apoyo a las balanzas de pago, sumados a los organismos financieros multilaterales, pueden contribuir significativamente a facilitar el ajuste de las cuentas externas de los países y evitar así efectos desproporcionadamente adversos al comercio intrarregional.

Sin embargo, con la excepción del FLAR (Fondo Latinoamericano de Reservas), los mecanismos de apoyo a las balanzas de pagos han carecido de la capacidad financiera necesaria para responder a una demanda generalizada de recursos. De la experiencia acumulada con esos mecanismos se deduce que, para que efectivamente contribuyan a resolver los desequilibrios de la balanza de pagos, en el marco de la escasez de recursos externos regionales a largo plazo, deben poseer una organización y personalidad jurídica propias y estar dotados de medios suficientes para satisfacer las demandas.

\subsection{El manejo de los desequilibrios} regionales.- Cuando se producen desequilibrios macroeconómicos transitorios pueden aparecer desfases comerciales capaces de generar fuertes tensiones en un proceso de integración incipiente. Es por eso que podrían surgir demandas en el sentido de corregir desequilibrios bilaterales aún antes de que se hayan creado las condiciones y acumulado la experiencia necesaria para establecer un procedimiento de coordinación, y sin que existan mecanismos financieros regionales o suficientes recursos para enfrentar tal problema. Se trata de una situación compleja, porque exige a los países 
corregir desequilibrios de los flujos intrarregionales de comercio aún cuando presumiblemente ninguna de las partes esté dispuesta a modificar su propio manejo macroeconómico.

Ante una situación de este tipo se plantean disyuntivas. Si no se ha previsto una medida de flexibilidad desde el comienzo, la adhesión al proyecto de integración se presenta como una opción entre "todo o nada", la aparición de grandes desfases, tanto a nivel macroeconómico como de determinadas empresas, puede llevar el proyecto a la crisis . Alternativamente, un manejo demasiado flexible del proceso de integración comercial enrarece el clima para la adopción de decisiones sobre intercambio e inversión, dado que vuelve incierta la continuidad de las señales. Dejar las políticas en materia de comercio regional al arbitrio de las fluctuaciones cíclicas de precios y volúmenes no tiene sentido, excepto como solución de emergencia, a falta de otros instrumentos. Pero si se reconoce la existencia de esa posibilidad, sería importante definir pautas de antemano a fin de determinar qué acciones se considerarán aceptables.

\subsection{Sistemas de pago y financiamiento} de las exportaciones.- Los sistemas de pago pueden desempeñar un papel fundamental en la gestión de un regionalismo abierto. En principio, la función principal de las cámaras de compensación es reducir el número de transacciones bilaterales, lo que permite ahorrar tiempo y recursos. No obstante, también podrían contribuir a disminuir la incertidumbre que entraña el comercio, particularmente la probabilidad de que los agentes en el país de destino no salden sus compromisos. Es previsible que, a diferencia de las exportaciones tradicionales, que suelen ser realizadas por conducto de intermediarios de gran envergadura, las nuevas exportaciones intrarregionales se dirijan hacia compradores de menores dimensiones, lo que posiblemente incremente los riesgos conexos.

Cuando los sistemas de pago permiten reducir el uso de divisas convertibles internacionalmente, su utilidad disminuye a medida que se restablecen los mercados privados de divisas y las monedas nacionales se tornan más atractivas como medio de pago, lo que ha sido demostrado por la experiencia de la Unión Europea de Pagos. Pero si las monedas nacionales no son ampliamente utilizadas como medio de pago en América Latina, cobran mayor importancia los instrumentos de creación de liquidez regional que resulten más atrayentes desde el punto de vista de su convertibilidad, liquidez, estabilidad de valor y rentabilidad. El acceso a mecanismos que permitieran crear liquidez externa en la región podría moderar los efectos de fluctuaciones en la disponibilidad de recursos financieros internacionales y así reducir la vulnerabilidad del comercio intrarregional.

Dada la necesidad de insertar las empresas nacionales, particularmente las pequeñas y medianas, en el proceso de globalización y regionalización en curso, correspondería facilitar su articulación, entre otros medios, dotándolas de un acceso expeditivo al financiamiento. Actualmente la banca comercial desempeña el principal papel en lo que respecta al financiamiento de las exportaciones en los países de la región, pero el acceso al mismo está seriamente limitado. 
En la región, el avance hacia una amplia complementación del financiamiento bancario (y la promoción) de las exportaciones es un proceso reciente. Por ahora, sólo en algunos países se han previsto fondos de garantía (previas y posteriores al embarque), seguros de crédito para la exportación y otros mecanismos de financiamiento que aseguran un amplio acceso a los exportadores directos e indirectos, así como a la pequeña y mediana empresa. que permitan financiar las exportaciones de bienes de capital o la inversión en capital fijo necesario para exportar. La creación de sistemas amplios y expeditos para el financiamiento de las exportaciones no tradicionales podría representar un gran potencial para los países de la región y ejercer un efecto particularmente importante sobre las exportaciones intrarregionales.

\section{2.- BILATERALISMO Y MULTILATERALISMO COMERCIAL}

\subsection{La experiencia comercial regional.-}

En América Latina, la experiencia de los países centroamericanos en materia de acuerdos bilaterales en el pasado fue positiva, aunque de corta duración. Por una parte, se concertó una serie de acuerdos bilaterales de libre comercio intra-centroamericanos durante la década de los cincuenta, pero muy pronto fueron convertidos en un acuerdo multilateral que, posteriormente, facilitó la dinámica expansión del comercio intracentroamericano registrada durante los años sesenta y setenta. Por otra parte, los procesos de industrialización y liberalización del comercio al interior de la subregión se dieron simultáneamente, lo que hizo que los acuerdos bilaterales y, más tarde, los subregionales, enfrentaran menos obstáculos resultantes de intereses creados al amparo de políticas nacionales de sustitución de importaciones.

Los miembros de la ALALC y la ALADI, en cambio, tuvieron una experiencia menos fructífera. Por una parte, se estancó el proceso de liberalización promovido en el contexto de la ALALC mediante la multilateralización de concesiones comerciales negociadas bilateralmente. En ese debilitamiento incidió el hecho de que durante la etapa de sustitución de importaciones, la protección nacional otorgada a los procesos de industrialización dio orígenes a intereses creados opuestos a la liberalización amplia de mercados a nivel nacional. Esa actitud, sumada a las tentativas de regulación vinculadas al esfuerzo de programación industrial a nivel de la subregión, contribuyó a frenar la expansión del libre comercio intrarregional en el marco del Pacto Andino.

Por otra parte, al percibir el grado de rigidez de la ALALC, se intentó dar mayor flexibilidad al procesode integración mediante un fortalecimiento de su dimensión bilateral, lo cual llevó a la constitución de la ALADI, con la firma del tratado de Montevideo en 1980. Al autorizar la suscripción de acuerdos de alcance parcial, el tratado facilitó el bilateralismo no sólo en el ámbito de las concesiones comerciales, sino también en el de las normas aplicables al comercio intrazonal.

Así, dos factores que reflejan parcialmente las distintas posiciones de los gobiernos en cuanto a la posibilidad de multilateralizar los esquemas bilaterales, contribuyeron a obstaculizar la convergencia entre el bilateralismo y 
la extensión multilateral (regional) de los acuerdos convenidos por los miembros de la ALADI durante la década de los ochenta.

Primero, su armonización se hizo depender básicamente de instancias institucionales multilaterales, cuyas facultades habían sido cuestionadas o reducidas.

Segundo, sin que hubieran normas expresas al respecto, se tendió a supeditar la convergencia de los acuerdos entre los miembros de la ALADI a la paulatina desaparición de los convenios de alcance parcial y a su sustitución progresiva y facultativa por los de preferencia arancelaria y otros acuerdos de alcance regional. En un marco de desequilibrios y de ajustes que hacían más fácil restringir las importaciones cuando existían acuerdos bilaterales, era poco probable que se diera este tipo tan amplio de convergencia.

\subsection{Adhesión efectiva a los acuerdos.- En} el actual contexto de apertura comercial, la adhesión flexible y efectiva a los acuerdos preferenciales a otros países sería congruente con la gestación de un regionalismo abierto en cuyo marco se redujera la discriminación intrarregional. Promover la adhesión efectiva ofrece dos ventajas. Por una parte, reduce la superposición de áreas, lo que provoca la multiplicidad de acuerdos simultáneos de integración; y puede facilitar la constitución de grupos de países que actuen como propulsores de procesos renovados de integración regional.

Por otra parte, la flexibilidad de incorporación reduce la probabilidad de desviación de comercio y hace más factible el aprovechamiento de economías de escala; también puede incidir positivamente en la inversión. Lo anterior supone privilegiar, mediante procesos flexibles de adhesión, la liberalización amplia del comercio entre países que ya realizan un nutrido intercambio (socios naturales).

La heterogeneidad de las situaciones comerciales en la región, así como el hecho de que las relaciones bilaterales entre países de distinto tamaño pueden entrañar grandes diferencias en cuanto a la importancia relativa de los copartícipes, determinan que no haya ninguna relación bilateral en la que las proporciones del comercio recíproco sean superiores a 25 por ciento para ambos países. En otras palabras, se asume que esa proporción indica que dos países son socios naturales. Existen dos maneras de encarar esta situación. La primera sería establecer acuerdos preferenciales entre varios países de la región. para así aumentar la proporción de comercio efectivo sujeto a tratamiento preferencial. La segunda sería esperar que la liberalización comercial de carácter unilateral que ya se ha producido genere gradualmente una mayor interdependencia comercial. Solamente entonces, en una etapa ulterior, se suscribirían acuerdos preferenciales basados en el razonamiento de los socios naturales.

\section{3.- AEC (ARANCELES EXTERNOS COMUNES ).}

\subsection{Papel del AEC en el proceso de} integración.- El establecimiento de un arancel externo común representa un esfuerzo por profundizar, más que por ampliar, un acuerdo de integración. Por consiguiente, su factibilidad será menor en un contexto de progresivo aumento del número de miembros de un acuerdo que en uno de consolidación de compromisos ya adquiridos. A su vez, la conveniencia de establecer el AEC dependerá de las 
características de los países que suscriben un acuerdo de integración. Suponiendo que sus niveles sean bajos, los aranceles comunes se justificarán más en el caso de países que combinen un alto grado de comercio recíproco y estructuras económicas similares. En estos casos, la aplicación de aranceles diferenciales a los diversos insumos introduciría desigualdades en los niveles de protección efectiva de cada país miembro y generaría condiciones propicias para prácticas desleales y el establecimiento de restricciones al comercio.

Por otra parte, las diferencias de precios de los productos importados del resto del mundo incentivarían el contrabando. El arancel externo común también facilita la realización de negociaciones comerciales conjuntas, si bien éstas no exigen su existencia. Además, reduce la necesidad de imponer normas de origen estrictas $y$, por consiguiente, de introducir medidas proteccionistas adicionales, puesto que se elimina el incentivo para ingresar importaciones por conducto del país con aranceles más bajos. Una alternativa sería adoptar aranceles comunes de manera gradual, dando prioridad a aquellos sectores en donde las diferencias de incentivos provocan mayores distorsiones.

$3.2 \mathrm{El}$ TLC y los aranceles comunes.Aunque en el TLC (Tratado de Libre Comercio) no se contempla un arancel externo común, se adoptó excepcionalmente en el caso de las computadoras. Esta medida facilitó llegar a un acuerdo sobre normas de origen relativamente sencillas, ya que al no existir aranceles externos diferentes tampoco hay incentivo para introducir computadoras en un país miembro del
TLC por conducto del que tenga menores aranceles. En el curso de diez años, México reducirá su arancel externo, que actualmente fluctúa entre 10 y 20 por ciento, a los niveles aplicados por Estados Unidos y Canadá, que están entre 3.7 y 3.9 por ciento. También se eliminarán dentro de un plazo de siete años, los beneficios del reintegro de derechos (drawback) a los que pueden acogerse las industrias maquiladoras, que producen la mayor parte de las computadoras fabricadas en México. La regla de origen establece simplemente que las computadoras serán consideradas norteamericanas si la tarjeta de circuitos es elaborada en la región y si su transformación se realiza de tal manera que se modifica su clasificación arancelaria.

\subsection{Normas que rigen la competencia} desleal y el Acuerdo General de Aranceles y Comercio (GATT).- La legislación sobre derechos compensatorios, antidumping y salvaguardias constituye un complemento de la política arancelaria, en especial cuando se está desarrollando un proceso de apertura que, al eliminar la protección excesiva, aumenta la posibilidad de abusos derivados de prácticas desleales de comercio. A su vez, los procedimientos para enfrentar tales prácticas tienden a ser complejos en términos de metodología, de medición y de organización. Es por eso que difícilmente pueden imaginarse las dificultades jurídicas y administrativas y las incertidumbres de los agentes económicos que causaría la proliferación de acuerdos de integración con diferentes disposiciones sobre prácticas de comercio desleal.

Por otra parte, el origen de las controversias en el ámbito de las 
relaciones comerciales internacionales ha tendido notoriamente a radicarse en disposiciones acerca de las barreras no arancelarias. En este sentido, resaltan las referidas a la aplicación de las cláusulas sobre subsidios y derechos compensatorios, y sobre derechos compensatorios y antidumping.

Los procedimientos para dirimir tales diferencias generalmente contemplan la constitución de grupos arbitrales una vez agotadas las instancias previas de consulta o mediación.

Una forma de reducir la complejidad y los costos asociados a la mayor o menor discriminación que puede resultar de diversos acuerdos de integración es restringir al mínimo el número de instrumentos de política comercial (aranceles y reglas de origen) que puedan generar efectos preferenciales como consecuencia de negociaciones bilaterales o subregionales. Para lograrlo se puede recurrir a códigos multilaterales, como los del GATT, y aplicarlos a la regulación de otros instrumentos de política comercial. En este sentido, resulta particularmente evidente la complementariedad entre el avance de las negociaciones en el ámbito multilateral (GATT) y el de las tratativas regionales y subregionales de carácter preferencial. En la medida en que se logren avances en el ámbito del GATT sobre temas como antidumping, subvenciones, derechos compensatorios y salvaguardias, se podrá aplicar a nivel nacional, subregional o regional, sin necesidad de adoptar compromisos discriminatorios frente a terceros. Se evitarían así engorrosas negociaciones bilaterales o subregionales en torno de cada uno de esos temas.

Por otra parte, el GATT constituye una instancia supranacional que puede complementar o sustituir la capacidad institucional que requieren los acuerdos de integración.

\section{4.- POTENCIALIDADES DE LA INTEGRACIÓN ECONÓMICA}

\section{I El rol que puede jugar la} integración.- Los escenarios internacional y regional han variado sustancialmente desde que nosotros (Herrera.1982) planteamos la necesidad y conveniencia de que los países de América Latina emprendieran un proceso de integración económica. En el panorama actual, la integración debe exhibir características distintas, porque sus propósitos tienen que ajustarse a una nueva realidad.

Lo que se quiere es reducir la vulnerabilidad de los países latinoamericanos frente al entorno internacional, a la globalización, disponiendo de relativa autonomía para diseñar y aplicar sus propias políticas económicas con la finalidad de cambiar cualitativamente su inserción en la economía mundial. La profundización del proceso de integración y cooperación permitirá organizar y ejercer un poder de negociación mayor dentro del plano internacional.

El rol en los inicios de los procesos de integración era el desarrollo sistemático de la industrialización, por ser considerado el motor del desarrollo integrador. El tamaño de los mercados internos definía el tipo de actividades productivas que se podían implementar de manera autónoma, y aquellas que por sus economías de escala excedían las potencialidades del espacio doméstico. En este caso, la integración de los mercados aparecía como la única fórmula que hacía posible su desarrollo eficiente. 
En los casos de los países pequeños y medianos, la integración económica viabilizaba una industrialización selectiva, en cambio, para los países grandes, hacía factible un rango más amplio de actividades productivas.

En la actualidad, el tamaño de las fábricas productoras continúa jugando un rol decisivo para lograr costos competitivos en determinadas ramas económicas; sin embargo, el desarrollo tecnológico y la tercera revolución industrial imponen un tipo de instalación industrial más versátil y "reducida". Lo anterior no significa que los países no tengan acceso a la creación de industrias "gigantes" (siderurgia, petroquímica, del aluminio, de bienes de capital y de electrónica avanzada), sino que la principal limitación se encuentra en la capacidad tecnológica y productiva del conjunto de la economía para potenciar y brindar la infraestructura necesaria de apoyo a las empresas productoras.

En general, en los países de América Latina no existe una infraestructura que sea capaz de sustentar el desarrollo autónomo. La principal carencia se encuentra en la capacidad científica y tecnológica, y en su potencia] para colaborar en la modernización y la transformación permanente de la capacidad productiva mediante el empleo de tecnologías que incorporan más valor intelectual y más conocimientos, en contraposición a la mano de obra no calificada.

Esta realidad tiende a disminuir una de las ventajas que tiene la región: la abundancia de mano de obra de bajo costo relativo. En los países desarrollados, el rápido crecimiento de servicios altamente especializados y de elevada tecnología se realiza a partir de sistemas productivos "maduros", en un proceso simbiótico de mutua alimentación.

La transformación desde una especialización internacional basada en actividades intensivas en mano de obra a otra de mayor contenido tecnológico no es un proceso simple ni automático. El incremento de los recursos aplicados al desarrollo científico y tecnológico y a la innovación, debe siempre estar acompañado de un poderoso sistema de capacitación y de formación de personal altamente capacitado y especializado.

Por otro lado, el modelo de sustitución de importaciones se fundamentaba principalmente en cada mercado doméstico, no resultando lo selectivo y profundo que era menester para crear una estructura productiva completa y diversificada. De este modo no pudo lograrse un desarrollo más autosostenido, que generara los medios suficientes para adecuarla a los cambios que se experimentaban en el ámbito internacional.

De este modo, un mercado ampliado resultante de un proceso de integración efectivo puede ayudar a crear las condiciones de un desarrollo tecnológico y productivo más acorde con una participación activa en la economía internacional, así como viabilizar desenvolvimientos conjuntos que precisan de grandes espacios económicos de sustentación.

En el complejo marco de profundas mutaciones en la economía mundial- derivadas del progreso científico y tecnológico y la "internacionalización" o "globalización" de los procesos productivos- la noción de mercado ampliado difiere del concepto tradicional. 
En efecto, éste se entendía preferentemente como el resultado de mecanismos de liberalización arancelaria y de la eliminación de las restricciones para-arancelarias, en un plano multilateral.

El mercado ampliado se concibe como un medio para producir un sólido enfrentamiento entre las economías y sus operadores (sean éstos públicos o privados) en el cual el elemento central está dado por la capacidad de concertación entre gobiernos para impulsar diversos esquemas de producción e iniciativas de inversión compartidas.

En este modelo. la concertación comprende acuerdos y mecanismos de desgravación arancelaria y la eliminación de otras restricciones al comercio, pero el aspecto modular se encuentra en la formación de una sólida y estable interdependencia económica y política entre los países participantes en cada esquema de integración.

En resumen, la evolución económica y productiva de las últimas décadas exige concepciones e integración y cooperación diferentes a las tradicionales. En ellas, los instrumentos, especialmente los de carácter arancelario, pierden importancia relativa y deberá privilegiar la búsqueda de una interdependencia económica más estable y global.

\subsection{La unidad regional y subregional.-}

Después de la Segunda Guerra Mundial pareció florecer un período de cooperación internacional, ensombrecido por la denominada "guerra fría" EsteOeste. La crisis internacional desatada en la década de los ochenta ha puesto de relieve que en las relaciones internacionales se ha acentuado el uso de la fuerza y la presión como normas de convivencia. Sin embargo, a pesar de la caída del Muro de Berlín (Nov.1989) éstas prácticas no han cambiado, sino que, por el contrario, se han profundizado y refinado.

Así, el proteccionismo de los países desarrollados, ejercido principalmente por Japón, la Unión Europea y los Estados Unidos (los socios comerciales más importantes de América Latina), hace indispensable crear un poder de negociación que ayude a neutralizar los perniciosos efectos que estas acciones podrían generar en los principales rubros de exportación regional.

La unidad latinoamericana es un requisito para el desarrollo de una estrategia de modernización y dinamización del sistema productivo. Asimismo, es igualmente condición necesaria para organizar y ejercer una capacidad de negociación en el plano internacional que tenga credibilidad y eficacia.

Todo ello supone que la industrialización, el desarrollo agrícola y del sistema productivo en general y sus servicios colaterales se apoyen prioritariamente en el mercado regional o subregional, según sea el caso. Este enfoque corresponde al convencimiento de que en las actuales circunstancias de la economía mundial, para América Latina es esencial aprovechar los potenciales de los mercados internos conjuntos, como una forma de aumentar la autonomía nacional y regional. En otras palabras, acrecentar los grados de libertad para elegir estrategias de desarrollo que permitan un mayor dominio sobre los recursos y las estructuras productivas y cambiar cualitativamente el modo de su inserción en la economía internacional. 
5.- COORDINACIÓN DE LAS POLÍTICAS MACROECONÓMICAS

5.1 La urgencia de este tipo de coordinación.- Un requisito de la coordinación, en cualquiera de sus formas, es que las partes manifiesten tanto una demanda como una oferta de acciones comunes. La integración comercial no solamente incrementa la demanda de coordinación al intensificar la transmisión de los efectos macroeconómicos, sino que también hace más estrecha que en el pasado la interacción entre las políticas de los países, a la vez que da mayor relieve a la evolución económica de las partes. Además, en la medida en que las aperturas unilaterales incrementen el comercio intrarregional, los países se verán envueltos en una integración macroeconómica más acentuada, sea que existan o no preferencias comerciales.

Ahora bien, la demanda de coordinación de cada país surge de la percepción de un posible beneficio en el caso de que los demás realicen una determinada acción. Esto excluye las situaciones en las que la interdependencia macroeconómica entre los países es débil, y también aquellas en que las dimensiones económicas son muy asimétricas.

Cuando existe un proyecto de integración, la coordinación macroeconómica puede enfocarse como una resultante o bien como uno de los cimientos para el desarrollo de intercambios, en la primera perspectiva. Lo importante es cómo organizar un manejo coordinado de los instrumentos de política de cada país que permita internalizar los efectos transmitidos desde cualquiera de las economías a las demás. Por otra parte, la demanda de coordinación tendería a aparecer en forma episódica, cuando las circunstancias alejen a los países de sus metas macroeconómicas.

Si bien ambos enfoques no son totalmente excluyentes, un examen de las iniciativas de coordinación puestas en la práctica permite distinguir entre las que corresponden principalmente a uno u otro tipo. Así, el Grupo de los Siete funciona en buena medida como un foro en el que las partes tratan problemas de política específicos, pero no ceden autonomía de decisión. En la Unión Europea, en cambio, la coordinación macroeconómica se ha planteado como un componente fundamental de la integración, por lo que se han formulado reglas institucionalizadas para el manejo de las diversas variables que eso involucra.

\subsection{Las condiciones iniciales que} favorecen la coordinación.- Cuando se emprende un ejercicio de coordinación con propósitos definidos, las partes pueden acordar un manejo concertado de determinados instrumentos, o bien fijar objetivos relacionados con el comportamiento de variables endógenas en cada país, dejando a las autoridades respectivas la elección de los medios para lograr los resultados previstos. En términos generales, la coordinación implica que las políticas de los países mantengan trayectorias análogas constantes: la respuesta conjunta a una perturbación puede requerir que los instrumentos de cada país actúen en direcciones opuestas.

Sin embargo, desde el punto de vista de la oferta de coordinación, la capacidad para ejecutar políticas de regulación macroeconómica ha estado severamente limitada en América Latina.

En particular, los instrumentos fiscales y monetarios en los que se centra, 
e.. la atención del Grupo de los Siete, en lo: países de la región están a menudo condicionados en exceso. En todo caso, suelen definirse más en respuesta a las presiones o urgencias del momento que a una programación precisa. Si bien la situación puede ser distinta en los países que han logrado una cierta normalización macroeconómica, en un análisis general de las posibilidades de coordinación en el ámbito latinoamericano no se pueden pasar por alto las restricciones en cuanto a la disponibilidad de instrumentos de política económica.

Las instancias de coordinación laxa serían útiles, además, para conciliar ideas sobre enfoques generales de política y tratar casos en los que exista el riesgo de que se produzcan acciones y reacciones contrarias a la cooperación (i.e., escaladas de medidas cambiarias o comerciales dirigidas contra otro país miembro), como resultado de divergencias en la evolución de las partes o de perturbaciones externas. Asimismo, un requisito para llegar a acuerdos concretos sobre el manejo de instrumentos es que cada participante conozca las metas, restricciones y enfoques de política de los demás, lo que es posibilitado por una interacción reiterada; ésta. bajo ciertas condiciones, también permite que surjan mecanismos propicios para incrementar la credibilidad entre las partes.

Aunque difícilmente se podría esperar que los países renuncien a utilizar instrumentos que consideran eficaces, una forma incipiente de coordinación -que tal vez precediera a la búsqueda de consensos sobre valores numéricos de variables de política-sería establecer, de común acuerdo, reglas generales sobre el tipo de políticas que adoptarían las partes. Este principio de compromiso presumiblemente asumiría la forma de un enunciado negativo, es decir, de medidas que no se deberían aplicar para no entorpecer las relaciones bilaterales.

En suma, un acuerdo genérico de corte cualitativo sobre las formas de manejar las políticas macroeconómicas facilita, y tal vez hace posible, la coordinación cuantitativa. Otra forma de establecer reglas comunes podría ser la implantación de un esquema en dos etapas, basado en un conjunto de indicadores; en la primera etapa se identificarían las carencias en materia de coordinación y, en la segunda, las medidas correctivas que se deberían adoptar.

\subsection{La coordinación de la política} cambiaria.- Dado que en los países o la región el mercado interno de deuda pública es generalmente limitado $\mathrm{y}$, en muchos casos, existe un considerable grado de movilidad internacional de capitales, la conducción monetaria se desenvuelve dentro de márgenes estrechos. De ahí que la tónica de la política macroeconómica suele ser determinada principalmente por los instrumentos fiscales y cambiarios. Por consiguiente, el espacio disponible para acciones de coordinación dependerá del grado de libertad para manejar esos instrumentos.

Sin embargo, la coordinación de la política cambiaria, que está vinculada con la de otras políticas macroeconómicas, enfrenta el fundamental problema de que no existe consenso sobre qué régimen cambiario sería el más idóneo para maximizar los beneficios netos de dicha coordinación.

El hecho de que, pese a los reparos hechos al régimen cambiario internacional, el Grupo de los Siete haya 
tomado como base la flotación "sucia" de las monedas, parece denotar dificultades para llegar a una convergencia de las políticas fiscales y monetarias en las grandes áreas económicas. Quizás también significa que se ha tenido en cuenta la noción implícita de que, si los precios relativos en esas áreas están sujetos a stocks reales, conviene que las paridades nominales puedan variar con flexibilidad para reducir los costos del ajuste.

Por otra parte, la participación en un régimen monetario común requiere que los miembros posean un alto grado de competitividad en cuanto a manejo presupuestario a largo plazo. Esto supone imponer fuertes restricciones a las políticas nacionales, especialmente durante el período de transición.

La fijación de la paridad cambiaria es una medida común en programas de estabilización, en especial -pero no exclusivamente- en aquellos que buscan hacer frente a procesos inflacionarios muy severos. Los regímenes de tipo de cambio fijo limitan estrictamente el manejo financiero del gobierno, y a menudo se aplican para dotar de un marco definido a la gestión fiscal y monetaria y reforzar las expectativas de los agentes. Al elegir una divisa extranjera como patrón monetario, el país generalmente busca aprovechar la mayor credibilidad relativa de su política monetaria que esa medida implica en el país en referencia.

Aunque es teóricamente concebible que varios países cuyo manejo de la moneda no sea estable puedan establecer un esquema monetario conjunto, sin incurrir en sesgos inflacionarios, los requisitos institucionales para que ello ocurra parecen demasiado estrictos como para considerarla una opción real. Por lo tanto, sería de esperar que, si se produjera una convergencia de políticas cambiarias entre países tradicionalmente inestables en materia macroeconómica, el esquema no considere una flotación conjunta, sino la vinculación de las monedas a una o más divisas centrales. Sin embargo, aún así sería necesario que el destino y origen de su comercio fuera semejante.

Además, como lo sugieren las recientes turbulencias monetarias, puede ser difícil mantener indefinidamente paridades fijas, aún entre países con organizaciones macroeconómicas similares.

\section{CONCLUSIONES}

El espíritu menos formalista y de mayor realismo que demuestran los esquemas más recientes de integración en el hemisferio indica que se están reconociendo ciertos principios básicos. Parece que en el origen de esta tendencia existe una aceptación del hecho de que las instituciones deben responder adecuadamente a las características del proceso de integración que se pretenda desarrollar. Así, si sólo se trata de abolir los obstáculos a la libre circulación de mercancías, las instituciones del esquema deberán ser predominantemente intergubernamentales y relativamente modestas.

A su vez. cuando el objetivo sea la creación de una moneda común, se deberá establecer por lo menos un sistema integrado de bancos centrales o un banco central, como en el caso de la estructura de la reserva federal. Si la iniciativa apunta a la coordinación de políticas, es evidente que se debe buscar una medida de centralización de los poderes de decisión que posibilite la puesta en práctica de 
políticas industriales o agrícolas comunes, o bien el manejo coordinado de los indicadores con el fin de aplicar una política macroeconómica conjunta.

En todo caso, lo más aconsejable sería evitar la adopción de compromisos rígidos y favorecer los esquemas dotados de la adaptabilidad necesaria como para que sea la propia dinámica del proceso la que imponga el ritmo del desarrollo institucional. De esta manera, la forma reflejará la función y las instituciones cumplirán su principal cometido, que es el de ser a la vez instrumentos de estabilidad y de cambio.

Finalmente, si se pretende que las instituciones respondan a valores y a la cultura actuales de América Latina, tienen que reflejar el vuelco hacia la democratización y la representatividad que se manifiesta en todo el hemisferio. Esto significa que se deben permitir y facilitar mayores niveles de participación de las organizaciones representativas de los diversos intereses sociales. En el mismo orden de ideas, cabría apoyar las instancias comunes, tales como el Parlamento Latinoamericano, que permitan articular los procesos políticos al mismo tiempo que la integración económica.

En la medida en que aumenta la participación de las organizaciones no gubernamentales se hace más complejo el proceso de adopción de decisiones, ya que para dar oportuna cabida a las opiniones de los nuevos actores se deben crear los mecanismos adecuados. Si realizan este esfuerzo, las instituciones de los procesos de integración adquirirán una mayor legitimidad, al aumentar su capacidad para responder a las aspiraciones de los diversos grupos sociales.

\section{BIBLIOGRAFIA}

CEPAL

1992. Equidad y Transformación Productiva: Un Enfoque Integrado.

CEPAL

1994. América Latina: Políticas para mejorar su inserción en la economía mundial.

DORNBURSCH R.,

1993. North-South trade relations in the Americas: The case for free trade.

FRENCH-DAVIS, R., 1993. Beneficios y costos de la integración comercial.

HALPERIN M., 1992. Instrumentos básicos de Integración económica en América Latina.

HERRERA GARCIA, Beatriz. 1982. Grupo Andino y Comecon. Crítica a la Integración Económica.

HERRERA GARCIA, Beatriz. 1988. Teoría del Comercio Internacional y de la Integración Económica.

HEYMANN, D. y F. NAVAJA.

1992. Aspectos conceptuales de la coordinación de políticas macroeconómicas con referencia al Mercosur. Ensayos sobre coordinación de políticas macroeconómicas: Inferencias para la Integración latinoamericana.

IIEc-UNAM.

1996. Problemas del Desarrollo. En: Revista Latinoamericana de Economía Nros. 104 y 108

INTAL

1974. Análisis de los márgenes de preferencia en el comercio intrarregional 
en el marco de la Asociación Latinoamericana de Libre Comercio.

ONU

1990-1991. Suplemento del Estudio Económico Mundial. En: Departamento de Desarrollo Económico y Social. Nueva York, 1992.

ROSENTAL, Gert 1994. "América Latina y el Caribe frente a la economía mundial". En:. Revista de la Cepal. Nro. 53.

RUBIATO, J.M.

1993. Acuerdos múltiples en el transporte internacional. Propuestas de criterios y mecanismos de negociación. Santiago de Chile.

SCHERER, P.M. y D. ROSS.

1990. Industrial Market Structure and Economic Perfomance. H. Mifflin. Boston.

SELA.

1992. The relationship between competition policy and trade policy: an introduction to the issues.

Caracas.

\section{SELA.}

1993. Normas de origen: análisis económico y propuestas de acción multilateral.

\section{Caracas.}

SINGER, H.W., 1992. ¿ Es posible una genuina asociación en una zona de libre comercio en el hemisferio occidental?. Algunos comentarios generales. Documentos de trabajo sobre comercio en el hemisferio occidental.

Washington, D. C.

ZELADA CASTAÑEDO, A.,

1983. "Convergencia y multilateralismo en ALADI". Documento presentado al seminario "El Cono Sur de América Latina: Nuevas perspectivas de cooperación regional".

Chile. 